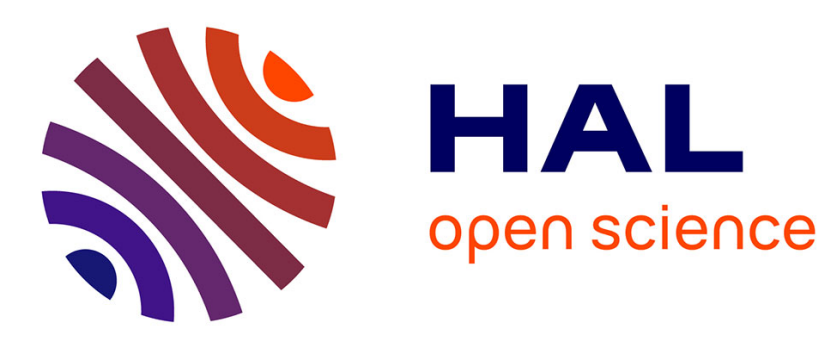

\title{
La Sicile fatimide, plaque tournante du commerce maritime entre le monde musulman et l'Europe
}

David Bramoullé

\section{To cite this version:}

David Bramoullé. La Sicile fatimide, plaque tournante du commerce maritime entre le monde musulman et l'Europe. Héritages arabo-islamiques dans l'Europe méditerranéenne, 2015, 978-2-7071-8622-5. hal-01536398

\section{HAL Id: hal-01536398 \\ https://hal.science/hal-01536398}

Submitted on 11 Jun 2017

HAL is a multi-disciplinary open access archive for the deposit and dissemination of scientific research documents, whether they are published or not. The documents may come from teaching and research institutions in France or abroad, or from public or private research centers.
L'archive ouverte pluridisciplinaire HAL, est destinée au dépôt et à la diffusion de documents scientifiques de niveau recherche, publiés ou non, émanant des établissements d'enseignement et de recherche français ou étrangers, des laboratoires publics ou privés. 


\section{La Sicile fatimide, plaque tournante du commerce maritime entre le monde musulman et l'Europe}

David Bramoullé*

$\mathrm{D}$

$\mathrm{u}$ début du $\mathrm{x}^{\mathrm{e}}$ siècle au milieu du XI $\mathrm{I}^{\mathrm{e}}$ siècle, la Sicile forma l'une des provinces de l'Empire fatimide. Ce califat, fondé vers 909 au Maghreb, fut transféré sur les rives du Nil sous le règne d'al-Mu'izz li-Dîn Allâh (953-975), quatrième calife de la dynastie. Les califes fatimides, d'obédience chiite, s'attachèrent dans tous les domaines à concurrencer le califat sunnite des Abbassides considérés comme illégitimes. L'opposition se fit autant militaire qu'idéologique et économique. Dans ce domaine, les Fatimides voulaient surpasser l'aura commerciale de Bagdad, considérée comme «l'entrepôt du monde ", au profit de Fostat et du Caire, capitales économiques et politiques de l'Égypte. Toutes les marchandises du monde connu devaient donc converger vers l'Égypte et tous les territoires fatimides, du Maghreb au Yémen, furent mis à contribution. La Sicile joua alors un rôle essentiel.

À la tête de l'île depuis 937, les émirs kalbides encouragèrent l'essor des productions artisanales et agricoles et développèrent une brillante vie de cour pour les besoins de laquelle il fallait importer des quantités de produits orientaux, synonymes de luxe et de raffinement. Ainsi, en

* $\quad$ Maître de conférences, université Toulouse Jean Jaurès. FRAMESPA. UMR 5136. 
dépit de l'éloignement des califes du Caire et des rapports ambigus avec les Zirides de Mahdia, la Sicile garda des liens commerciaux privilégiés tant avec l'Ifrīqiya et qu'avec l'Égypte. Si cette dernière pouvait apparaître comme la plaque tournante majeure du commerce entre l'Orient et l'Occident, le voyage vers Alexandrie, principal port fatimide sur la Méditerranée, n'était pas accessible à tous les marchands. Les risques du trajet, ainsi que les difficultés et les frais du séjour, pouvaient en décourager plus d'un.

La Sicile disposait donc de nombreux atouts pour devenir une place centrale alternative du commerce entre le monde musulman et l'Europe, comme en témoigne la documentation et notamment les lettres commerciales judéo-arabes de la Geniza - un dépôt documentaire du Vieux Caire - dans lesquelles la Sicile jouit d'une place privilégiée. Les divers documents mettent clairement en évidence le développement d'une agriculture et d'un artisanat de qualité, mais aussi et surtout les liens commerciaux de plus en plus étroits tissés entre la Sicile, l'Ifrīqiya et l'Égypte, ainsi qu'avec l'Europe du sud située à quelques encablures.

\section{La Sicile, un centre de productions réputées et exportées}

Loin du tableau dressé en 973 par le géographe Ibn H̦awqal qui rapportait que les intérêts vitaux des Siciliens dépendaient des importateurs, les productions agricoles étaient au contraire nombreuses et donnaient lieu à des exportations réputées [Ibn Hawqal, 1967, p. 122]. L'exportation de produits alimentaires vers l'Ifrīqiya est attestée dès le $\mathrm{x}^{\mathrm{e}}$ siècle, alors que les Fatimides s'y trouvaient encore. Il semble toutefois que les productions et les exportations s'amplifièrent au siècle suivant. Une lettre, écrite à Mahdia en 1061, atteste le rôle majeur que l'île jouait alors pour l'approvisionnement de l'ancienne capitale fatimide. Labrât b. Moshe b. Sughmâr, juge de la communauté juive de Mahdia écrivait alors Nahray b. Nissîm, à propos de Mahdia, « cette ville est dépendante [de la Sicile] pour la nourriture » et Labrât d'évoquer avec inquiétude la hausse des prix que la progression des Normands en Sicile provoquait sur les marchés locaux [Simonsohn, 1997, p. 302].

$S^{\prime}$ il ne dit rien des produits alimentaires qui provenaient de Sicile, la documentation permet de s'en faire une idée. Parmi les productions 
agricoles, les céréales occupaient l'essentiel des terres de Sicile. Il s'agissait principalement d'orge et de froment, notamment du blé dur à partir duquel al-Idrîsî atteste qu'à l'époque normande au moins on fabriquait des vermicelles (itrîyah) près de Palerme [Idrîsî, 1999, p. 309]. Le riz de Sicile était également exporté vers l'Égypte et sans doute aussi le Maghreb, comme en atteste une lettre de 1060 [Simonsohn, 1997, p. 291]. La conquête musulmane permit également d'introduire ou de développer un certain nombre de cultures grâce à l'importation de nouvelles techniques agraires. Ainsi, la canne à sucre, introduite dans la région de Palerme et mentionnée pour la première fois vers 930, se diffusa dans l'île. Les califes fatimides, dont les cuisines produisaient de grandes quantités de pâtisseries, faisaient une grande consommation de sucre. Ils possédaient des plantations de cannes dans l'île et le sucre candi figure parmi les exportations siciliennes au $\mathrm{X}^{\mathrm{e}}$ siècle, mais la tendance s'inversa nettement ensuite, sans doute à cause de la consommation palatiale [Ibn Hawqal, 1967, p. 122].

Les vergers de Sicile et la qualité des fruits qui y poussaient ont également constitué une forme de topos de la littérature médiévale [Bresc, 1972]. Si les agrumes jouissaient d'une grande réputation partout en Méditerranée, les textes évoquent surtout l'exportation de jus de fruits, mais aussi de vin comme en atteste une lettre écrite à Mazara del Vallo et envoyée à Fostat vers 1060 [Simonsohn, 1997, p. 295]. Les châtaignes, les noix, les noisettes et les amandes, exportées décortiquées (lawz maksûr) constituaient également des produits largement exportés. L'élevage permettait la fabrication d'un fromage très réputé en Égypte, ainsi qu'un artisanat du cuir. Les peaux de bœuf de Syracuse sont par exemple citées et des cargaisons de centaines de peaux de bœuf, mais aussi de daim, apparaissent dans les textes [Simonsohn, 1997, p. 27 et 126]. Enfin, la pêche au thon et à l'espadon se pratiquait tout autour de l'île [Idrîsî, 1999, p. 310-312]. Le poisson était exporté sous forme de salaisons vers l'Ifrīqiya et peut-être aussi vers la Provence [Treglia et al., 2012].

Les matériaux stratégiques faisaient également partie des exportations de l'île. La construction navale constituait une activité majeure du califat fatimide dans laquelle la Sicile jouait un rôle majeur. Au nom du jihâd, les Fatimides multiplièrent les raids maritimes d'Alexandrie à Alméria, en passant par Otrante, Gênes, la Corse ou encore Reggio [Bramoullé, 2009]. Les forêts siciliennes, notamment la zone 
chrétienne du Val Demone, furent abondement exploitées. Le bois d'œuvre, la poix mais aussi le fer des mines de Sicile étaient exportés vers Mahdia, le principal arsenal de la dynastie. Ibn Hawqal souligne la grande qualité du papyrus produit dans l'île. Si la majeure partie servait à confectionner les documents nécessaires à l'administration locale, l'autre partie était tressée pour fabriquer les câbles des ancres de navires [Ibn Hawqal, 1967, p. 121-122].

Plusieurs plantes destinées à l'artisanat textile furent également introduites en Sicile à l'époque fatimide. L'introduction du coton semble ainsi s'être produite vers le $x^{e}$ siècle. Des plantations se trouvaient notamment dans les régions d'Agrigente et de Mazara. Signalée dès la période byzantine, la culture du mûrier à ver à soie connut aussi un certain essor à partir du $\mathrm{x}^{\mathrm{e}}$ siècle. Ces plantes permirent le développement d'un artisanat textile de qualité. Le tissage de vêtements de coton et de soie est attesté à Palerme, Syracuse, Demone, et dans la région d'Agrigente. Les lettres de la Geniza signalent les nombreuses soieries produites dans l'île. Il s'agissait de diverses pièces de tissus, ainsi que plusieurs types de robes, de couvertures ou encore de foulards, mais aussi de tapis ou bien de matelas sergés en soie de très grande qualité. Très réputés, ces matelas se retrouvent jusqu'au Yémen et en Inde. Leur prix, entre cinq et dix dinars pièce, en faisait des produits de luxe que même l'imâm de la principale mosquée du Caire, la mosquée al-Azhar, payé environ trois dinars par mois au début du $\mathrm{XI}^{\mathrm{e}}$ siècle, pouvait difficilement s'offrir. Les textes évoquent aussi une soie de qualité inférieure, le lâsin, exportée en masse. Enfin, le gentilhomme oriental se devait de porter un turban en soie de Sicile, pour lequel on n'hésitait pas à dépenser deux fois plus que pour le vêtement principal [Goitein, 1971].

Les diverses productions siciliennes jouissaient donc d'une grande réputation et leur exportation contribuait à l'accroissement du trafic maritime entre la Sicile et le reste de la Méditerranée.

\section{Un contexte favorable au commerce}

L'essor des productions siciliennes et du trafic commercial qui passait par l'île ne doit rien au hasard. Le contexte local, et plus largement méditerranéen, était globalement favorable au commerce. Dès le IXe siècle, la multiplication des raids vers les villes italiennes et l'afflux 
d'or en provenance du «pays des Noirs» (Bilâd al-Sudân) favorisa la monétarisation de l'économie sicilienne et la frappe de quarts de dinars très prisés, qui facilitaient grandement les échanges. D'autre part, les villes de Sicile connurent une croissance notable. Palerme fut progressivement dotée de nouveaux quartiers. Un «quartier des Slavons » et un "nouveau quartier» (hârat al-Jadîda) firent leur apparition. L'émir Ja'far (998-1019) s'inspira du Caire fatimide pour lotir un quartier à son nom, al-Ja'fariyya [Bagnera, 2013, p. 61-88]. Dans la littérature arabe et judéo-arabe, Palerme apparaît souvent comme «la ville» (al-madîna) ou la «ville de Sicile» (madînat Siqîliyya), signe de son poids politique et économique dans l'île [Ben Sasson, 1991, p. 237, 365]. Les artisans et le marché liés aux consommations de la Cour y étaient nombreux. Dans les campagnes, il existait de nombreuses fermes qui appartenaient aux princes ainsi que des domaines agricoles, propriétés distribuées aux hauts fonctionnaires sans doute dès le $\mathrm{X}^{\mathrm{e}}$ siècle. D’une manière générale, le contexte méditerranéen était celui d'un essor du trafic maritime de plus en plus contrôlé par les cités italiennes intéressées par les profits qu'elles pouvaient tirer de la revente de produits orientaux qui arrivaient en nombre en Égypte d'abord, mais également de plus en plus en Sicile où les élites locales tenaient à suivre la mode orientale.

L'intensité des relations économiques et humaines entre la Sicile et l'Égypte apparaît de manière concrète dans de nombreuses lettres commerciales. Nahray b. Nissîm, l'un des plus célèbres marchands de la communauté juive de Fostat et destinataire de la lettre de Labrât en 1061, s'inquiétait lui-même de l'évolution des conditions politiques de l'île. Son propre frère opérait à Mazara et, à l'instar de nombreux marchands d'Égypte, il faisait davantage affaire avec la Sicile et l'Ifrīqiya qu'avec les ports de Syrie-Palestine, pourtant plus proches de l'Égypte. L'étude sérielle des lettres de la Geniza témoigne de la place centrale que jouait la Sicile dans l'économie égyptienne. Les trajets reliant la Sicile à l'Égypte et l'Égypte à la Sicile étaient plus fréquents que les trajets entre le Maghreb et l'Égypte. Les lettres rédigées en Sicile et destinées à Alexandrie ou Fostat sont plus nombreuses que les lettres rédigées en Ifrīqiya [Nef, 2007]. Nasir Khusraw confirme ce rôle lorsqu'il signale les liens commerciaux entre Alexandrie et la Sicile, mais ne dit rien de l'Ifrīqiya [Khusraw, 2001, p. 55]. La Sicile constituait la principale base de départ et d'arrivée des vaisseaux commerciaux qui commerçaient entre l'Égypte et l'espace siculo-tunisien. Le 
trafic maritime entre la Sicile et l'Ifrīqiya consistait semble-t-il davantage en une concentration de produits ifrîqiyens en Sicile avant l'exportation vers l'Égypte ou, au contraire, en un trafic de redistribution des produits importés dans l'île. Ainsi, les navires quittaient généralement les côtes maghrébines chargés des meilleures productions ifrîqiyennes vers les ports de Sicile dont la hiérarchie évolua au cours de la période.

Le contexte sicilien, de plus en plus instable, ne pénalisa pas le commerce mais favorisa une réorganisation du réseau portuaire sicilien. Des ports comme Trapani et surtout Mazara del Vallo virent leur rôle se renforcer au détriment de Palerme [Goldberg, 2012, p. 319]. Avec son «marché des vendeurs de lin » (sûq al-qattânîn), Mazara, au sud de l'île, devint le port principal pour les navires effectuant le trajet entre la Sicile et l'Égypte. Le lin brut égyptien était exporté en grande quantité vers l'île, où il était travaillé et réexporté. Les marchands égyptiens guettaient avec anxiété les nouvelles indiquant le prix du lin à Mazara. Cela leur permettait d'ajuster les exportations afin de ne pas faire s'effondrer les cours même si la demande était forte. Une lettre de 1063 envoyée à Fostat signale le manque de lin, mais aussi d'épices sur les marchés siciliens. L'auteur fait notamment part à son interlocuteur de son espoir de voir arriver « un gros navire en provenance de Syrie» [Simonsohn, 1997, p. 332]. Le courrier met en évidence que parvenaient en Sicile non seulement du lin, mais aussi les marchandises issues du commerce à travers l'océan Indien. De grandes quantités d'épices - comme le poivre, la cannelle et le gingembre - mais aussi de substances tinctoriales indispensables aux textiles - comme le bois-brésil et l'indigo -, ou encore de produits odoriférants et pharmaceutiques - tels le bois d'aloès, l'essence de rose, le myrobolan, la laque et le camphre - étaient chargées dans les cales des navires en partance pour la Sicile.

Des centaines de bâtiments faisaient les trajets chaque saison de et vers la Sicile. Une lettre de 1056 signale par exemple l'arrivée à Alexandrie de dix bateaux en provenance de Sicile portant chacun 500 personnes [Simonsohn, 1997, p. 194]. Un tel convoi n'avait alors rien d'exceptionnel. Les noms des propriétaires des navires sont tous cités et permettent de comprendre que l'île se trouvait en fait au cœur d'un réseau commercial reliant l'ensemble du monde musulman d'al-Andalus jusqu'à la Syrie, voire bien au-delà. 


\section{Une île au cour d'un vaste réseau commercial}

Au carrefour des deux bassins de la Méditerranée et à quelques encablures à la fois des terres chrétiennes et musulmanes, la Sicile jouait en réalité un rôle majeur non seulement pour le commerce et la navigation entre l'est et l'ouest de la Méditerranée, mais aussi entre les rives nord et sud. Au regard des quantités de produits de luxe importés en Sicile, il paraît assez évident que tous ces produits n'étaient pas uniquement destinés à une consommation locale, mais qu'une partie était réexportée vers al-Andalus ou le Maghreb ainsi que vers l'Europe chrétienne. La proximité de l'île avec les terres chrétiennes, les liens historiques et politiques de certaines communautés siciliennes avec Constantinople favorisaient aussi la présence de marchands venus de la péninsule italienne. L'île agissait comme un véritable $h u b$ moderne et ses ports servaient d'emporium pour les navires syriens, égyptiens ou maghrébins mais aussi et pour ceux qui étaient originaires d'al-Andalus et d'Italie.

Si quelques-uns des dix navires mentionnés en 1056 appartenaient à des armateurs siciliens, un était propriété de l'émir de Dénia et deux sont clairement désignés comme "Shâmî ", c'est-à-dire originaires de Syrie-Palestine. Ils appartenaient à Ibn 'Ammâr et Ibn Abî 'Aqîl, qui exerçaient tous deux les fonctions de cadis-gouverneurs de Tripoli du Liban et de Tyr. Profitant de leurs fonctions, ces deux hommes avaient chacun leur propre flottille commerciale qu'ils louaient aux marchands. Le calife al-Mustansir Billâh (1036-1094) possédait luimême des navires de commerce basés à Tripoli de Syrie qui effectuaient des trajets réguliers vers le Maghreb et la Sicile [Khusraw, 2001, p. 17]. La présence de navires orientaux dans les eaux siciliennes n'avait donc rien d'exceptionnel, mais les navires siciliens se retrouvaient aussi à mouiller dans les ports syriens.

En outre, les lettres de la Geniza signalent des bâtiments d'al-Andalus attendant le départ dans le port de Palerme où résidaient des marchands originaires de la péninsule Ibérique. À Palerme, ces derniers se trouvaient très clairement au centre d'un vaste réseau commercial et avaient accès à beaucoup plus de marchandises, et sans doute aussi de clients potentiels, que s'ils étaient demeurés à Almería ou Dénia. On note ainsi des chargements de sulfure, de sel-ammoniaque, de bois-brésil au départ de la Sicile vers al-Andalus, tandis que 
la soie et les cuirs d'al-Andalus étaient débarqués en Sicile ou réexportés vers l'Orient [Constable, 1994, p. 157, 174 et 208].

La proximité de la Sicile avec la Péninsule italienne facilita également les contacts entre les deux territoires. Dès le IX siècle, les marchands italiens se pressaient déjà en Sicile et en Ifrīqiya pour fournir un certain nombre de produits alimentaires, mais aussi du bois, du fer et des esclaves. Ils repartaient avec de l'huile d'olive, de la cire ainsi que de l'or, qui parvenait en quantité en Ifrīqiya et en Sicile depuis le Bilâd al-Sudân [Citarella, 1968]. L'installation des Fatimides en Ifrīqiya fit néanmoins franchir un cap aux relations commerciales entre leurs territoires et les cités italiennes, particulièrement Amalfi dont l'autonomie à l'égard de Constantinople lui permettait de commercer des productions qui semblent avoir été interdites à Venise par exemple. Ainsi, au xe siècle, les besoins des Fatimides en matériaux stratégiques se renforcèrent considérablement. Amalfi devint alors le fournisseur essentiel des Fatimides en bois et en fer, car les ressources endogènes ne suffisaient plus. Ce rôle permit à la cité d'échapper aux nombreux raids fatimides contre les cités italiennes et conféra à ses marchands une place privilégiée une fois les Fatimides installés en Égypte [Skinner, 2013, p. 223 ; Cahen, 1954, p. 3-8]. Les Amalfitains fréquentèrent de manière assidue les ports fatimides d'Ifrīqiya, de Sicile puis ceux d'Égypte après 973. La place centrale de la Sicile transparaît également dans certains documents qui montrent clairement que les Amalfitains arrivaient souvent en Égypte avec, dans leurs cales, des marchandises siciliennes. L'île était donc clairement une étape pour tous ceux qui faisaient du commerce en Méditerranée centrale. Au cours du XI siècle, la perspective de trouver en Sicile un certain nombre de productions locales et orientales encouragea d'autres cités comme Pise, Naples ou encore Venise, et peut-être aussi les Provençaux, à fréquenter les ports siciliens [Heyd, 1885, p. 122].

La présence de marchands dits Rûm - au Xie siècle, le terme désigne en général les marchands originaires d'Italie du Sud, et notamment d'Amalfi - apparaît très peu dans les textes arabes. En revanche, elle est clairement attestée par la documentation judéo-arabe. Une lettre rédigée à Trapani vers 1053 évoque la vente de poivre et peut-être aussi de laque à des Rûm. En 1056, apprenant que des marchands étrangers cherchaient à éviter de payer certains droits en concluant des accords avec les locaux qui payaient des taxes plus faibles à l'importation, l'émir imposa une dîme aux étrangers (ghurbâ') [Simonsohn, 1997, 
p. 137, 184 et 227]. Cette mesure, vécue comme une véritable injustice par les marchands de la Geniza, concernait en fait tous les étrangers et pas seulement les marchands juifs non siciliens [Nef, 2007]. Elle témoigne de la régularité de la présence de marchands non siciliens dans l'île. Une autre lettre, rédigée cette fois à Mazara en septembre 1064, confirme ces informations et apporte quelques détails importants : "L'an dernier, je suis allé à Palerme après l'arrivée des navires. J'y ai récupéré un ballot de poivre à l'intérieur duquel j'ai trouvé un paquet de gingembre noir de très mauvaise qualité. Par Dieu ! Personne ici ne m'en prendra la valeur d'un dirham, ni les Rûm ni personne d'autre [...]» et, plus loin, l'auteur de préciser que pour arriver à vendre son gingembre, il dut se résoudre à conclure un partenariat avec un collègue qui se rendait au "pays des Rûm» [Simonsohn, 1997, p. 332]. Le naturel avec lequel l'auteur évoque les ventes aux Rûm en dit long sur leur présence à Palerme. La lettre met aussi en évidence que dans certains cas, les marchands de la Geniza se rendaient dans les villes italiennes. Un document de 1065 signale ainsi l'hivernage à Salerne d'un marchand [Gil, 1997, p. 72]. Ces lettres sont néanmoins plus rares que celles attestant l'arrivée de Rûm.

Il ne fait en tout cas guère de doute que si de nombreux marchands européens se rendaient directement en Égypte pour obtenir les marchandises souhaitées à de meilleurs prix, les risques et les coûts étaient également plus grands. Tous n'avaient sans doute pas le courage ou l'argent pour effectuer une traversée qui variait de 20 à 50 jours de mer depuis l'Italie. Les ports siciliens constituaient des espaces commerciaux alternatifs très intéressants pour des commerçants européens qui ne pouvaient effectuer le voyage en Orient. Ainsi, une partie non négligeable sans doute des importations siciliennes depuis l'Égypte étaient achetées par des commerçants étrangers et notamment les Italiens, qui fréquentèrent de manière sans doute plus intense que les quelques textes conservés ne l'indiquent, les ports de Sicile afin de se procurer des produits de plus en plus appréciés dans une Europe alors en pleine croissance économique.

En dépit d'un contexte sicilien de plus en plus troublé, la Sicile vit son rôle de plaque tournante alternative du commerce entre l'Orient et l'Occident se renforcer. La position idéale de l'île au centre de la Méditerranée, le développement d'une cour soucieuse de consommer des produits de luxe ainsi que les liens privilégiés avec Le Caire favorisèrent les productions locales et surtout les importations des meilleurs 
produits issus du monde musulman. Un tel contexte rendait le voyage en Sicile plus que rentable pour certains marchands italiens qui évitaient ainsi le périple long et dangereux jusqu'à Almería, Alexandrie ou Tyr. La certitude de s'y procurer presque en voisins des marchandises très demandées en Europe compensait sans doute le prix ou encore le choix peut-être moindre voire la qualité inférieure de certaines marchandises par rapport à celles qu'il aurait été possible de trouver en Égypte. La construction de l'État normand ne modifia que peu le rôle de la Sicile, même si de plus en plus de chrétiens se rendirent directement à Alexandrie au XII siècle.

\section{Références bibliographiques}

BAgNERA A. (2013), «From a small town to a capital : the urban evolution of islamic Palermo ", in Nef E. (dir.), A Companion to Medieval Palermo. The History of a Mediterranean City from 600 to 1500, Leyde, Brill, p. 61-88.

BEN SASSON M. (1991), Yehudei Sitsilia, Jerusalem, Ben Zvi Institute.

BRAmoullé D. (2009), « Activités navales et infrastructures maritimes ; les éléments du pouvoir fatimide en Méditerranée orientale (969-1171) », in FAbre G., Le Blevec D., Menjot D. (dir.), Les Ports et la navigation en Méditerranée au Moyen Âge, actes de colloque (Lattes, 12-14 novembre 2004), Paris, Éditions Le Manuscrit, p. 253-270.

Bresc H. (1972), « Les jardins de Palerme (1990-1460) », Mélanges de l'école française de Rome, 54, p. 55-127.

CAHEN Cl. (1954), « Un texte peu connu relatif au commerce oriental d'Amalfi au Xe siècle », Archivio Storico per le Province Napoletane, 34, p. 3-8.

Citarella A. O. (1968), « Patterns of medieval trade, the commerce of Amalfi before the Crusades », Journal of Economics History, 28, p. 531-555.

Constable O. R. (1994), Trade and Traders in Muslim Spain, Cambridge, Cambridge University Press.

Gil M. (1997), In the Kingdom of Ishmael, vol. 3, Tel Aviv-Jérusalem, Tel Aviv University-Bialik Institute.

GoITEIN S. D. (1971), « Sicily and southern Italy in the cairo geniza documents ", Archivio storico per la Sicilia Orientale, 67, p. 9-33.

Goldberg J. L. (2012), Trade and Institutions in the Medieval Mediterranean, Cambridge, Cambridge University Press. 
La Sicile fatimide, plaque tournante du commerce maritime entre le monde...

Heyd W. (1885), Histoire du commerce du Levant au Moyen Âge, Leipzig-Paris, Harraszowitz-Lechevauer.

IBN HAWQAL (1964), Configuration de la Terre, t. 1, Paris, Maisonneuve et Larose.

IDRISI (1999), Première géographie de l'Occident, trad. H. BRESC et A. NeF, Paris, Flammarion.

Khusraw N. (2001), Books of Travels, éd. et trad. M. Thackston, Costa Mesa, Mazda Publishers.

Nef A. (2007), « La Sicile dans la documentation de la Geniza cairote (fin $\mathrm{X}^{\mathrm{e}}$-XIII ${ }^{e}$ siècle) : les réseaux attestés et leur nature », in Coulon D., PicARd Ch., Valérian D. (dir.), Espaces et réseaux en Méditerranée, $V I^{e}-X V I^{e}$ siècle, t. 1 : La configuration des réseaux, Saint-Denis, Bouchène, p. 273-292.

Simonsohn S. (1997), The Jews in Sicily, Leyde, Brill.

SKINNER P. (2013), Medieval Amalfi and Its Diaspora, 800-1250,

Oxford, Oxford University Press.

Treglia J.-C. et al. (2012), « Importations d'Amphores médiévales dans le sud-est de la France (Xe-XII ${ }^{e}$ s.) », in GeLICHI S. (dir.), Actes du $I X^{e}$ Congrès international de l'Association internationale pour l'étude des céramiques médiévales et modernes (Venise, 23-29 novembre 2009), p. 205-207. 